\title{
Electronic structure and superconductivity of $\mathrm{MgB}_{2}$
}

\author{
D M GAITONDE, P MODAK*, R S RAO and B K GODWAL \\ High Pressure Physics Division, Bhabha Atomic Research Centre, Mumbai 400 085, India
}

\begin{abstract}
Results of $a b$ initio electronic structure calculations on the compound, $\mathbf{M g B}_{2}$, using the FPLAPW method employing GGA for the exchange-correlation energy are presented. Total energy minimization enables us to estimate the equilibrium volume, $c / a$ ratio and the bulk modulus, all of which are in excellent agreement with experiment. We obtain the mass enhancement parameter by using our calculated, $D\left(E_{\mathrm{F}}\right)$ and the experimental specific heat data. The $T_{\mathrm{c}}$ is found to be $37 \mathrm{~K}$. We use a parametrized description of the calculated band structure to obtain the $T=0 \mathrm{~K}$ values of the London penetration depth and the superconducting coherence length. The penetration depth calculated by us is too small and the coherence length too large as compared to the experimentally determined values of these quantities. This indicates the limitations of a theory that relies only on electronic structure calculations in describing the superconducting state in this material and implies that impurity effects as well as mass renormalization effects need to be included.
\end{abstract}

Keywords. Electronic structure; superconductivity; magnesium diboride; coherence length; London penetration depth.

\section{Introduction}

The discovery of superconductivity (Nagamatsu et al 2001), with remarkably high transition temperature, in the binary compound, $\mathrm{MgB}_{2}$, with $T_{\mathrm{c}} \sim 39 \mathrm{~K}$ has initiated extensive studies of this compound. At ambient conditions, $\mathrm{MgB}_{2}$ crystallizes in the layered hexagonal $\mathrm{AlB}_{2}$ type structure, where $\mathrm{B}$ atoms form a primitive honeycomb lattice consisting of graphite-like sheets separated by hexagonal layers of $\mathrm{Mg}$ atoms. Boron isotope effect (Bud'ko et al 2001) has been observed in $\mathrm{MgB}_{2}$ giving credence to the belief that the pairing mechanism leading to superconductivity is of phononic origin. On substituting $\mathrm{Al}$ for $\mathrm{Mg}$ it is found (Slusky et al 2001) that $T_{\mathrm{c}}$ decreases. Another intriguing feature of this compound is that spectroscopies which probe the superconducting gap have reported conflicting results. Thus some measurements have seen (Karapetrov et al 2001; Takahashi et al 2001) a single gap, whereas others have measured (Chen et al 2001; Szabo et al 2001) two different gaps. The inplane penetration depth in this compound is measured (Niedermayer et al 2001) to be $\sim 1000 \AA$ whereas the coherence length is found (de Lima et al 2001) to be $70 \AA$.

A natural question that arises is whether studies of the electronic band structure of this material combined with BCS theory or its strong coupling variant can account for the superconducting state properties of this compound. Motivated by this we have carried out $a b$ initio electronic structure calculations using the full potential linear augmented plane wave method (FPLAPW) employing the

\footnotetext{
*Author for correspondence
}

generalized gradient approximation (GGA) (Perdew et al 1996) for the exchange-correlation energy. We then proceed to minimize the total energy and determine the cla ratio from which the equilibrium volume is calculated. We also calculate the bulk modulus of this material. All of these quantities are found to be in excellent agreement with experiments.

We next turn our attention to the superconducting properties. Motivated by the isotope effect seen by Bud'ko et al (2001), we worked within the BCS model and its strong coupling generalization. We used the McMillan (1968) formula for strong coupling superconductivity to estimate the value of $T_{\mathrm{c}}$. We adopted the parametrized description proposed by Kong et al (2001) of the boron $\sigma$ and $\pi$ bands which occur near the Fermi surface to calculate the $T=0 \mathrm{~K}$ penetration depth and superconducting coherence length. This calculation was performed separately for the one and two gap situations. Our estimate of $T_{\mathrm{c}}$ yielded a value of $37 \mathrm{~K}$, very close to the experimental value of $39 \mathrm{~K}$. However, the penetration depth calculated by us was too small by a factor of ten, whereas the coherence length was too large by a factor of four. Both these results indicate the inadequacy of electronic band structure in providing a detailed description of the superconducting properties of $\mathrm{MgB}_{2}$. It is very likely that a correct description of the superconducting state requires the inclusion of impurity effects in addition to mass renormalization of the electrons at the Fermi surface.

In $\$ 2$ below we describe the calculations of the electronic band structure, equilibrium volume and cla ratio, bulk modulus and $T_{\mathrm{c}}$. In $\S 3$ we present the calculations of the zero temperature penetration depth and coherence length. Finally in $\$ 4$ we conclude by summarizing the 
main results of our work and discussing shortcomings that indicate directions for future work.

\section{Electronic structure calculations}

We have performed the $a b$ initio electronic structure calculation using FPLAPW as implemented in the WIEN97 code (Blaha et al 1990). In our methods we do not use the shape approximation for the potential as both spherical and non-spherical components of the potential have been taken into account in obtaining the radial solutions of the Schrödinger equation inside the muffin-tin spheres. The exchange-correlation terms in the KohnSham equation have been treated within the GGA. In our calculations the muffin-tin sphere radii for $\mathrm{B}$ and $\mathrm{Mg}$ are 1.45 a.u. and 2 a.u., respectively. For the interstitial region we have used 370 plane waves. The Brillouin zone sampling is done by taking 5000 k-points with $144 \mathbf{k}$ points being chosen in the irreducible wedge.

We calculate and minimize the total energy with respect to the $c / a$ ratio as well as the volume. Here $a$ and $c$ are the lattice parameters of $\mathrm{MgB}_{2}$ which crystallizes in the hexagonal $\left(\mathrm{AlB}_{2}\right)$ structure. We obtain the equilibrium volume to be 194.08 a.u. as compared to the experimental value (Nagamatsu et al 2001) of 196.04 a.u. The cla ratio obtained by us is 1.151 as compared to the experimental value (Nagamatsu et al 2001) of 1.142. We have interpolated the calculated total energy as a function of volume thus getting a smooth function whose derivatives we take to obtain pressure and the bulk modulus, B, which is found to be $148 \mathrm{GPa}$ which compares well with the measured (Garg et al 2001; Vogt et al 2001) value of $152 \mathrm{GPa}$ found from high pressure X-ray diffraction studies. The bulk modulus value determined by us is used to determine the sound velocity, $c_{\mathrm{s}}$, which in turn enables us to estimate the Debye temperature, $\boldsymbol{\theta}_{\mathrm{D}}$. We find $\boldsymbol{\theta}_{\mathrm{D}}=733 \mathrm{~K}$, whereas the experimental values mentioned for $\theta_{\mathrm{D}}$ are $750 \mathrm{~K}$ (Bud'ko et al 2001) and $1050 \mathrm{~K}$ (Bouquet et al 2001). We thus find that the structural properties of $\mathrm{MgB}_{2}$ are well described by electronic band structure.

The electronic band structure calculated by us shows that the bands near the Fermi energy are predominantly due to boron orbitals. There are four bands which cross the Fermi level thus giving rise to a multi-sheeted Fermi surface for this compound. The bands are plotted in figure 1. Two of the bands arise from the $\pi$ bonding and antibonding combinations of the $B-p_{\mathrm{z}}$ orbitals whereas the other two bands have their origin from the obonding combinations of the $B-s p^{2}$ hybrid orbitals. It is seen from figure 1 that the $\sigma$ bands are degenerate and nearly flat along $\Gamma-A$ direction. These flat bands largely contribute to the density of states near the Fermi level, thus making a high transition temperature possible. Our results are in agreement with those obtained earlier by Kortus et al

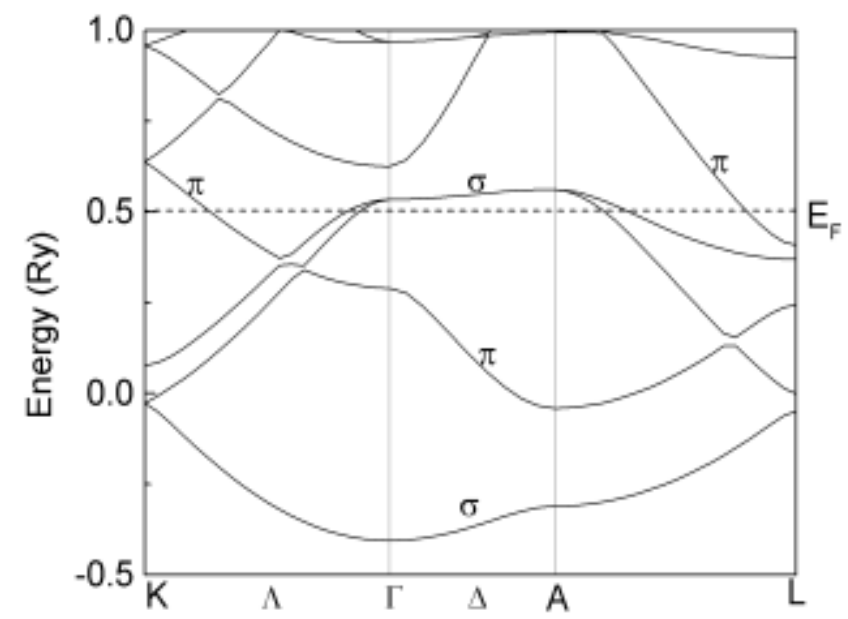

Figure 1. Band structure of $\mathrm{MgB}_{2}$ along some high symmetry directions.

(2001) and An and Pickett (2001), who also employed the FPLAPW method. Similar results have also been obtained in studies by Singh (2001) using pseudopotentials and plane waves.

We now describe our estimation of the superconducting transition temperature, $T_{\mathrm{c}}$. The calculated density of states at the Fermi level, $D\left(E_{\mathrm{F}}\right)$, enables us to calculate the bare electronic specific heat coefficient, $\gamma$ using the relation

$$
\gamma_{\text {cal }}=\left(\frac{1}{3}\right) \pi^{2} D\left(E_{\mathrm{F}}\right) k_{\mathrm{B}}^{2},
$$

which is found to be $1.7 \mathrm{~mJ} / \mathrm{mole}^{2} \mathrm{~K}^{2}$. The estimates made of $\gamma_{\text {exp }}$ from specific heat measurements vary from $2.6 \mathrm{~mJ} / \mathrm{mole}^{2} \mathrm{~K}^{2}$ (Bouquet et al 2001) to $3 \mathrm{~mJ} / \mathrm{mole}^{2} \mathrm{~K}^{2}$ (Bud'ko et al 2001). Thus, the mass enhancement factor $(1+\lambda)$ (Scalapino 1968) where $\lambda$ is the dimensionless electron-phonon coupling constant is determined using the relation

$$
(1+\lambda)=\gamma_{\text {exp }} / \gamma_{\text {cal }} \text {. }
$$

This yields the values, $\lambda=0.53$ and $\lambda=0.77$, corresponding to the two values of $\gamma$. We estimate $T_{\mathrm{c}}$ using the McMillan formula

$$
\begin{aligned}
T_{\mathrm{c}}= & \left(\boldsymbol{\theta}_{\mathrm{D}} / 1.45\right) \exp \{-1.04(1+\lambda) / \\
& \left.\left(\lambda-\mu^{*}(1+0.62 \lambda)\right)\right\},
\end{aligned}
$$

here $\theta_{\mathrm{D}}$ is the Debye temperature which is determined from lattice specific heat measurements. As mentioned earlier, it has been estimated to be $1050 \mathrm{~K}$ (Bouquet et al 2001) and $750 \mathrm{~K}$ (Bud'ko et al 2001). $\mu^{*}$ is the Coulomb pseudopotential whose value is chosen to be $0 \cdot 1$ as is conventional for $s$ and $p$ band superconductors. Thus the choice $\lambda=0.53$ and $\theta_{\mathrm{D}}=750 \mathrm{~K}$ yields $T_{\mathrm{c}}=9.4 \mathrm{~K}$. On the 
other hand choosing the most favourable parameters $\left(\lambda=0.77\right.$ and $\left.\theta_{\mathrm{D}}=1050 \mathrm{~K}\right)$, we obtain $T_{\mathrm{c}}$ to be $37 \mathrm{~K}$. In fact assuming the value of $\mu^{*}$, which is a poorly known quantity, to be 0.093 leads to a $T_{\mathrm{c}}$ of $39 \mathrm{~K}$ in this case. We note that we have not used our calculated $\theta_{D}$ to determine $T_{\mathrm{c}}$ as our calculation assumes an isotropic elastic medium which poorly describes the hexagonal $\mathrm{MgB}_{2}$ solid. The related compound, $\mathrm{AlB}_{2}$, has in comparison one extra electron per formula unit due to the replacement of divalent $\mathrm{Mg}$ by trivalent $\mathrm{Al}$. Within a rigid band picture, this is expected to move the Fermi energy away from the flat band feature leading to a depletion in the density of states at the Fermi energy. This provides a qualitative explanation of the reduction in $T_{\mathrm{c}}$ seen experimentally.

\section{Penetration depth and coherence length}

In order to derive the expression for the penetration depth we employ the semi-classical method. We introduce a magnetic field through a transverse vector potential, A. The band energies are then modified by the prescription

$$
\varepsilon_{n}(\mathbf{k}) \rightarrow \varepsilon_{n}(\mathbf{k}-2 \pi \mathbf{t} \mathbf{A} / h c)
$$

Here $n$ is the band index which runs from 1 to 4 , over the four bands that lie in the vicinity of the Fermi energy, $h$ the Plank's constant, $e$ the electronic charge and $c$ the velocity of light.

To a first order in A the BCS quasiparticle energies are Doppler shifted (Tinkham 1996) and are given by

$$
E_{n}(\mathbf{k})=E_{n}^{0}(\mathbf{k})-(2 \pi e \mathbf{A} / h c) \bullet \nabla_{k} \varepsilon_{n}(\mathbf{k}),
$$

where $E_{n}^{0}(\mathbf{k})=\left(\boldsymbol{\varepsilon}_{n}(\mathbf{k})^{2}+\Delta_{n}^{2}\right)^{1 / 2}$ is the quasiparticle energy in the absence of a magnetic field and $\Delta_{n}$ the energy gap which opens on the $n$th sheet of the Fermi surface.

The superconducting gap and BCS coherence factors are unchanged from their zero field values to the same order in $\mathbf{A}$. The current operator is then obtained by differentiating the electronic kinetic energy with respect to A and is found to be

$$
\begin{gathered}
J_{\alpha}=\left(2 \pi e / h N \Omega_{\mathrm{c}}\right) \sum_{n, \mathbf{k}, \sigma} \partial \varepsilon_{n}(\mathbf{k}) / \partial k_{\alpha} c_{\mathbf{k} \sigma}+c_{\mathbf{k} \sigma}-(2 \pi e / h)^{2} \\
\left(1 / c N \Omega_{\mathrm{c}}\right) \sum_{n, \mathbf{k}, \sigma, \mu} \partial^{2} \varepsilon_{n}(\mathbf{k}) / \partial k_{\alpha} \partial k_{\mu} c_{\mathbf{k} \sigma}+c_{\mathbf{k} \sigma}
\end{gathered}
$$

Here $\Omega_{\mathrm{c}}$ is the unit cell volume and $N$ the number of $\mathbf{k}$ points in the first Brillouin zone, the summation over $\sigma$ runs over up and down spins and $\alpha$ and $\mu$ are indices corresponding to the Cartesian components of the related vector.
We now evaluate the expectation value of the current operator, $\mathbf{J}$, in the BCS state to linear order in $\mathbf{A}$. We find that

$$
J_{\alpha}=-\rho_{\alpha \beta}^{\mathrm{s}} A_{\beta},
$$

where a summation over repeated indices is implied, and $\alpha$ and $\beta$ once again refer to Cartesian components.

$$
\begin{aligned}
\rho_{\alpha \beta}^{\mathrm{s}}=\left(8 \pi^{2} e^{2} / c h^{2} N \Omega_{\mathrm{c}}\right) & \sum_{n k}\left(g_{n}^{\alpha \beta}(\mathbf{k})+h_{n}^{\alpha \beta}(\mathbf{k})\right), \\
g_{n}^{\alpha \beta}(\mathbf{k})= & \left(\partial^{2} \varepsilon_{n}(\mathbf{k}) / \partial k_{\alpha} \partial k_{\beta}\right)\left[f\left(E_{n}^{0}(\mathbf{k})\right) \varepsilon_{n}(\mathbf{k}) / E_{n}^{0}(\mathbf{k})+0 \cdot 5\right. \\
& \left.\left(1-\varepsilon_{n}(\mathbf{k}) / E_{n}^{0}(\mathbf{k})\right)\right],
\end{aligned}
$$

and

$$
h_{n}^{\alpha \beta}(\mathbf{k})=\left[\partial \varepsilon_{n}(\mathbf{k}) / \partial k_{\alpha}\right]\left[\partial \varepsilon_{n}(\mathbf{k}) / \partial k_{\beta}\right] f\left(E_{n}^{0}(\mathbf{k})\right) .
$$

Here $\rho_{\alpha \beta}^{\mathrm{s}}$ is the superfluid stiffness tensor; $g_{n}^{\alpha \beta}(\mathbf{k})$ and $h_{n}^{\alpha \beta}(\mathbf{k})$ represent the diamagnetic response of the electronic condensate and the paramagnetic current of thermally excited quasiparticles and $f$ is the Fermi function. The London penetration depth, $\lambda_{\text {ex }}$, along the direction, $\alpha\left(\rho_{\alpha \beta}^{\mathrm{s}}\right.$ is found to be a diagonal tensor to a very good approximation) is given by $\lambda_{\alpha}^{-2}=4 \pi \rho_{\alpha \alpha}^{\mathrm{s}} / \mathrm{c}$.

To proceed further we use a parametrized form for the band energies proposed by Kong et al (2001). Our calculated band structure is in agreement with theirs thus justifying our use of their results. The two Tebands (antibonding and bonding) are parametrized as

$$
\begin{gathered}
\boldsymbol{\varepsilon}_{\pi}(\mathbf{k})=\varepsilon_{z}+2 t_{z}^{\perp} \cos \left(c k_{z}\right) \pm t_{z}\left(1+4 \cos \left(a k_{y} / 2\right)\right. \\
\left(\cos \left(a k_{y} / 2\right)+\cos \left(a k_{x} \sqrt{ } 3 / 2\right)\right)^{1 / 2} .
\end{gathered}
$$

The obands which are quasi 2-dimensional in character have Fermi surface sheets that are warped cylinders. The holes residing in these bands can therefore be described by the dispersion relation

$$
\boldsymbol{\varepsilon}_{\sigma n}(\mathbf{k})=\boldsymbol{\varepsilon}_{0}-2 t_{\mathrm{b}}^{\perp} \cos \left(c k_{z}\right)-k_{\mathrm{p}}^{2} / m_{n} .
$$

Here the index $n$ refers to the light (l) and heavy (h) hole bands, respectively, $k_{\mathrm{p}}$ the momentum component in the ab-plane and $m_{n}$ is a constant. All energies are with respect to the Fermi energy. The parameters occurring in these band descriptions are (Kong et al 2001) as follows: $\varepsilon_{\mathrm{z}}=0.04 \mathrm{eV}, \quad t_{z}^{\perp}=0.92 \mathrm{eV}, \quad t_{z}=1.6 \mathrm{eV}, \quad t_{\mathrm{b}}^{\perp}=0.094 \mathrm{eV}$, $\varepsilon_{0}=0.58 \mathrm{eV}, \quad m_{l}=0.07 \mathrm{eV}^{-1} \AA^{-2}$ and $m_{h}=0.15 \mathrm{eV}^{-1} \AA^{-2}$. The lattice constants are taken as $a=3 \cdot 105 \AA$ and $c=3.574 \AA$. The in plane $\sigma$ band wave vector, $k_{\mathrm{p}}$, is cut off at wave vector, $k_{\mathrm{BZ}}=2(2 \pi)^{1 / 2} / a(3)^{0.25}$, whose value has been chosen so as to preserve the Brillouin zone volume. 
Our calculations are performed at $T=0 \mathrm{~K}$ where the paramagnetic contribution to $\rho_{\alpha \beta}^{\mathrm{s}}$ vanishes due to the presence of a gap in the quasiparticle excitation spectrum. To evaluate the superfluid stiffness we introduce the functions

$$
\left.X_{\alpha \beta}^{n}(\omega)=1 / N \sum_{\mathbf{k}}\left(\partial^{2} \varepsilon_{n}(\mathbf{k}) / \partial k_{\alpha} \partial k_{\beta}\right) \delta \omega-\varepsilon_{n}(\mathbf{k})\right)
$$

The superfluid stiffness $\rho_{\alpha \beta}^{\mathrm{s}}$ is given by

$$
\begin{gathered}
\rho_{\alpha \beta}^{\mathrm{s}}=\left(8 \pi^{2} e^{2} / c h^{2} \Omega_{\mathrm{c}}\right) \sum_{n} \int \mathrm{d} \omega X_{\alpha \beta}^{n}(\omega)\left[f\left(\left(\omega^{2}+\Delta_{n}^{2}\right)^{1 / 2}\right)\right. \\
\left.\omega /\left(\omega^{2}+\Delta_{n}^{2}\right)^{1 / 2}+0 \cdot 5\left(1-\omega /\left(\omega^{2}+\Delta_{n}^{2}\right)^{1 / 2}\right)\right] .
\end{gathered}
$$

The k-sums in (7) are performed by taking a discrete grid of $30 \times 30 \times 20$ points in the Brillouin zone for the $\pi$ bands. In the case of the $\sigma$ bands, the $k_{\mathrm{p}}$ integrations can be done analytically and a numerical sum is needed only for the $k_{z}$ points. The $\omega$ integration in (8) is performed numerically using the Simpson rule method.

We do the calculation separately for one (Takahashi et al 2001) and two (Chen et al 2001) gap situations. In the former case we assume a uniform gap $\Delta(T=0)=5 \mathrm{meV}$ to have opened up over all four sheets of the Fermi surface whereas in the latter case following Golubov et al (2001) we take the larger gap $\Delta_{1}(T=0)=6.2 \mathrm{meV}$ to have opened over the $\sigma$ sheets and the smaller gap $\Delta_{\mathrm{s}}(T=0)=2.7 \mathrm{meV}$ to have opened up over the $\pi$ sheets.

We find that the zero temperature value of the penetration depth is insensitive to the one or two gaps scenarios. We obtain the in plane penetration depth, $\lambda_{\mathrm{ab}} \sim 87.35 \AA$, whereas the $c$-axis penetration depth is given by $\lambda_{c} \sim 310 \cdot 80 \AA$. These values are small by a factor of 10 as compared to the measured (Niedermayer et al 2001) values. To evaluate the coherence length we make use of the BCS relation (de Gennes 1968) for an isotropic superconductor $\xi=h v_{\mathrm{F}} / 2 \pi^{2} \Delta$, where $v_{\mathrm{F}}$ is the Fermi velocity and $\Delta$ the energy gap. We assume this relation to hold locally at each $\mathbf{k}$-point on the four Fermi sheets. We, therefore, calculate the Fermi surface average of $\left|\nabla_{k} \varepsilon_{n}(\mathbf{k})\right| / \pi \Delta_{n}$ on each of the four Fermi sheets. The coherence length is then obtained as a weighted sum of the lengths calculated on each sheet with weights $D_{n}(0) / D_{\text {tot }}(0)$. Here $D_{n}(0)$ is the density of states at the Fermi energy due to the $n$th band and $D_{\text {tot }}(0)$ is the total density of states at the Fermi energy. We find that the $T=0 \mathrm{~K}$ coherence length is different in the one-gap and two-gap situations. In the case of uniform gap we find $\xi \sim 255 \AA$ whereas in the presence of two gaps, $\xi \sim 377 \AA$. These values of the coherence length are larger by a factor of 4 to 5 from the coherence length extracted from upper critical field measurements.
The disagreement between our calculated values of $\lambda$ and $\xi$ and their measured values indicates the inadequacy of electronic band structure in describing the superconducting state properties of $\mathrm{MgB}_{2}$. Thus it seems that mass renormalization effects due to the strong electronphonon coupling believed to be present in this material need to be included. Also of importance are impurity effects which tend to increase the value of $\lambda$ and decrease the value of $\xi$ Thus our results are highly suggestive that $\mathrm{MgB}_{2}$ is not a clean superconductor.

\section{Conclusions}

In this work, we have calculated the electronic band structure of $\mathrm{MgB}_{2}$ using the FPLAPW method. Minimization of the total energy yields a c/a ratio, equilibrium volume and bulk modulus in excellent agreement with experiments. The band structure indicates that there are four bands near the Fermi energy giving rise to a multisheeted Fermi surface. Two of these bands originate from the $\pi$ bonding and antibonding orbitals derived from the boron $p_{z}$ atomic orbitals. The remaining two bands are derived from $\sigma$ bonding orbitals originating from the boron $s p^{2}$ hybrid orbitals involving the boron $p_{x}$ and $p_{y}$ orbitals. The obands are flat and nearly degenerate along $\Gamma-A$ direction. The largest contribution to the density of states near the Fermi energy comes from these flat bands and is therefore instrumental in achieving a high superconducting transition temperature. We estimate $T_{\mathrm{c}}$ from the McMillan formula to be $37 \mathrm{~K}$.

In order to verify the correctness of the electronic band structure derived picture in describing the superconducting state, we have calculated the zero temperature London penetration depth, $\lambda$ and coherence length, $\xi$ We derive an expression for the London penetration depth using a semi-classical description of the superconducting quasi-particles. The coherence length is obtained by performing a Fermi surface average of the BCS expression of the coherence length of an isotropic superconductor. We find that $\lambda$ calculated by us is smaller and $\xi$ larger than their experimentally determined values. This indicates that electronic structure on its own is inadequate in describing the superconducting properties of this compound and the agreement with experiment in the calculation of $T_{\mathrm{c}}$ may be fortuitous. Our calculations of $\lambda$ and $\xi$ provide a test of the applicability of DFT based band theory to describe the superconducting state properties of $\mathrm{MgB}_{2}$. Our results show that impurity effects, which increase $\lambda$ and decrease $\xi$ are probably very important and need to be included. Mass renormalization arising from the strong electronphonon coupling could also play an important role.

Finally we state some of the shortcomings of our work. The measurement of two different gaps implies that a theory of multi-band superconductivity needs to be 
worked out and our use of the one band McMillan formula to estimate $T_{\mathrm{c}}$ may not be justified. There are also indications from first principle phononic calculations (Liu et al 2001; Yildirim et al 2001) that there is a sizeable nonlinear electron-phonon coupling to a strongly anharmonic optic mode of $\mathrm{B}$ in plane vibrations. This would invalidate many results of conventional superconductivity theory based on the Migdal-Eliashbarg theory (Scalapino 1968) which treats linear electron-phonon coupling to harmonic acoustic modes.

\section{Acknowledgements}

We are thankful to Dr S K Sikka for several useful conversations and for having gone through the manuscript.

\section{References}

An J M and Pickett W E 2001 Phys. Rev. Lett. 864366

Blaha P et al 1990 Comput. Phys. Commun. 59399

Bouquet F et al 2001 Phys. Rev. Lett. 87047001

Bud'ko S L et al 2001 Phys. Rev. Lett. 861877

Chen X K et al 2001 preprint cond-mat/ 0104005
Garg A et al 2001 unpublished results

de Gennes P G 1966 in Superconductivity of metals and alloys

(New York: W. A. Benjamin Inc.) p. 225

Golubov A A et al 2001 preprint cond-mat/ 0111262

Karapetrov G et al 2001 Phys. Rev. Lett. 864374

Kong Y et al 2001 Phys. Rev. B64 020501(R)

Kortus J et al 2001 Phys. Rev. Lett. 864656

de Lima O F et al 2001 Phys. Rev. Lett. 865974

Liu A Y, Mazin I I and Kortus J 2001 Phys. Rev. Lett. 87 087005

McMillan W 1968 Phys. Rev. 167331

Nagamatsu J et al 2001 Nature 41063

Niedermayer Ch et al 2001 unpublished preprint

Perdew J P, Burke K and Ernzerhof M 1996 Phys. Rev. Lett. 77 3865

Scalapino D J 1968 in Superconductivity (ed.) R D Parks (New York: Marcel Dekker Inc.) Vol. 1, p. 522

Singh P P 2001 Phys. Rev. Lett. 87087004

Slusky J et al 2001 Nature 410343

Szabo P et al 2001 preprint cond-mat/0105598

Takahashi T et al 2001 Phys. Rev. Lett. 864915

Tinkham M 1996 in Introduction to superconductivity (New York: McGraw-Hill Inc.) p. 92

Vogt T et al 2001 preprint cond-mat/0102480

Yildirim T et al 2001 Phys. Rev. Lett. 87037001 